\title{
REMARKS ON THE RANK PROPERTIES OF FORMAL CR MAPS
}

\author{
BERNHARD LAMEL AND NORDINE MIR
}

\begin{abstract}
We prove several new transversality results for formal CR maps between formal real hypersurfaces in complex space. Both cases of finite and infinite type hypersurfaces are tackled in this note.
\end{abstract}

\section{INTRODUCTION}

Given two smooth real hypersurfaces $M, M^{\prime}$ in $\mathbb{C}^{N}$ and a smooth CR mapping $f: M \rightarrow M^{\prime}$, it is often of fundamental importance to know whether $f$ is CR-transversal at a given point $p \in M$, that is, whether the the normal derivative of the normal component of $f$ at $p$ is nonvanishing. This question naturally arises from regularity or unique continuation problems for local CR mappings between smooth or real-analytic hypersurfaces in complex space (see e.g. the works [20, 4, 10, 12, 7, 3, 13, 9).

In this note, we prove several new transversality results for CR mappings between real hypersurfaces in complex space. Our considerations being purely formal, we formulate our results in the context of formal CR maps between formal real hypersurfaces. Two opposite situations are tackled in this note. Firstly, when the source hypersurface $M$ is of finite type (in the sense of Kohn 14 and Bloom-Graham [8), we give a new sufficient condition on $M$ which guarantees that any (formal) holomorphic map sending $M$ into another (formal) real hypersurface in $\mathbb{C}^{N}$, with non-vanishing Jacobian determinant, is necessarily CR-transversal (Corollary 2.3). This result generalizes, in particular, a result due to Baouendi-Rothschild [5]. Secondly, we also deal with the case of infinite type hypersurfaces, which, to our knowledge, does not seem to have been much studied before (except implicitly in the works of Baouendi-Rothschild [6] and Meylan [19]). We prove, among other results, a sharp transversality result for self-maps of infinite type hypersurfaces (Corollary [3.3). We also provide, in such a setting, a new sufficient condition for a CR-transversal self-map to be an automorphism (Theorem 3.10).

In the next section, we collect the necessary notation and background needed in the paper. The results (and proofs) of the results in the finite type and in the infinite type case can be read independently and are given in $\$ 2$ and $\$ 3$ respectively.

\section{Notation AND PRELIMINARIES}

For $x=\left(x_{1}, \ldots, x_{k}\right) \in \mathbb{C}^{k}$, we denote by $\mathbb{C}[[x]]$ the ring of formal power series in $x$ and by $\mathbb{C}\{x\}$ the subring of convergent ones. If $I \subset \mathbb{C}[[x]]$ is an ideal and $F:\left(\mathbb{C}_{x}^{k}, 0\right) \rightarrow\left(\mathbb{C}_{x^{\prime}}^{k^{\prime}}, 0\right)$ is a formal map, we define the pushforward $F_{*}(I)$ of $I$ to be the ideal in $\mathbb{C}\left[\left[x^{\prime}\right]\right], x^{\prime} \in \mathbb{C}^{k^{\prime}}, F_{*}(I):=\left\{h \in \mathbb{C}\left[\left[x^{\prime}\right]\right]\right.$ : $h \circ F \in I\}$. We also define the generic rank of $F$, and denote it by $\operatorname{Rk} F$, to be the rank of the Jacobian matrix $\partial F / \partial x$ regarded as a $\mathbb{C}[[x]]$-linear mapping $(\mathbb{C}[[x]])^{k} \rightarrow(\mathbb{C}[[x]])^{k^{\prime}}$. Hence $\operatorname{Rk} F$ is

2000 Mathematics Subject Classification. 32H02, 32H12, 32V05, 32V15, 32V20, 32V25, 32V35, 32V40.

Key words and phrases. formal CR map, CR-transversality.

The first author was supported by the FWF, Projekt P17111. 
the largest integer $r$ such that there is an $r \times r$ minor of the matrix $\partial F / \partial x$ which is not 0 as a formal power series in $x$. Note that if $F$ is convergent, then $\mathrm{Rk} F$ is the usual generic rank of the map $F$. In addition, for any complex-valued formal power series $h(x)$, we denote by $\bar{h}(x)$ the formal power series obtained from $h$ by taking complex conjugates of the coefficients. We also denote by ord $h \in \mathbb{N} \cup\{+\infty\}$ the order of $h$ i.e. the smallest integer $r$ such that $\partial^{\alpha} h(0)=0$ for all $\alpha \in \mathbb{N}^{k}$ with $|\alpha| \leq r-1$ and for which $\partial^{\beta} h(0) \neq 0$ for some $\beta \in \mathbb{N}^{k}$ with $|\beta|=r$ (if $h \equiv 0$, we simply set ord $h=+\infty)$. Moreover, if $S=S\left(x, x^{\prime}\right) \in \mathbb{C}\left[\left[x, x^{\prime}\right]\right]$, we write $\operatorname{ord}_{x} S$ to denote the order of $S$ viewed as a power series in $x$ with coefficients in the ring $\mathbb{C}\left[\left[x^{\prime}\right]\right]$.

For $(Z, \zeta) \in \mathbb{C}^{N} \times \mathbb{C}^{N}$, we define the involution $\sigma: \mathbb{C}[[Z, \zeta]] \rightarrow \mathbb{C}[[Z, \zeta]]$ by $\sigma(f)(Z, \zeta):=\bar{f}(\zeta, Z)$. Let $r \in \mathbb{C}[[Z, \zeta]]$ that is invariant under the involution $\sigma$. Such an $r$ is said to define a formal real hypersurface through the origin, which we denote by $M$, if $r(0)=0$ and $r$ has non-vanishing gradient with respect to $Z$ at 0 . Throughout the note, we shall freely write $M \subset \mathbb{C}^{N}$. The complex space of vectors of $T_{0} \mathbb{C}^{N}$ which are in the kernel of the complex linear map $\partial_{Z} r(0)$ will be denoted by $T_{0}^{1,0} M$ (see e.g. [1] for the motivation of these definitions).

Throughout this note, it will be convenient to use (formal) normal coordinates associated to any formal real hypersurface $M$ of $\mathbb{C}^{N}$ (see e.g. 1]). They are given as follows. There exists a formal change of coordinates in $\mathbb{C}^{N} \times \mathbb{C}^{N}$ of the form $(Z, \zeta)=(Z(z, w), \bar{Z}(\chi, \tau))$, where $Z=Z(z, w)$ is a formal change of coordinates in $\mathbb{C}^{N}$ and where $(z, \chi)=\left(z_{1}, \ldots, z_{N-1}, \chi_{1}, \ldots, \chi_{N-1}\right) \in \mathbb{C}^{N-1} \times$ $\mathbb{C}^{N-1},(w, \tau)=(w, \tau) \in \mathbb{C} \times \mathbb{C}$ so that $M$ is defined through the following defining equations

$$
r((z, w),(\chi, \tau))=w-Q(z, \chi, \tau),
$$

where $Q \in \mathbb{C}[[z, \chi, \tau]]$ satisfies

$$
Q(0, \chi, \tau)=Q(z, 0, \tau)=\tau .
$$

In such a coordinate system, we now recall several well-known (invariant) nondegeneracy conditions for the formal real hypersurface $M$. Firstly, $M$ is said to be of finite type (in the sense of Kohn [14 and Bloom-Graham [8]) if $Q(z, \chi, 0) \not \equiv 0$. If $M$ is not of finite type, we say that $M$ is of infinite type. Following [18, 17, we say that $M$ belongs to the class $\mathcal{C}$ (resp. is essentially finite) if for $k$ large enough the formal (holomorphic) map $\chi \mapsto\left(Q_{z^{\alpha}}(0, \chi, 0)\right)_{|\alpha| \leq k}$ is of generic rank $N-1$ (resp. is a finite map). Recall also that $M$ is called holomorphically nondegenerate (in the sense of Stanton [21]) if the generic rank of the formal map $(\chi, \tau) \mapsto\left(Q_{z^{\alpha}}(0, \chi, \tau)\right)_{|\alpha|<k}$ is $N$. All these nondegeneracy conditions are known to be independent of the choice of normal coordinates. Note that if $M \in \mathcal{C}$, then $M$ is necessarily holomorphically nondegenerate. It is also good to point out for the purposes of this paper that formal real hypersurfaces that do not contain any formal curve are examples of hypersurfaces that belong to the class $\mathcal{C}$ (see [18, 17, 1] for more details).

If $r, r^{\prime} \in(\mathbb{C}[[Z, \zeta]])^{2}$ define two formal real hypersurfaces $M$ and $M^{\prime}$, we let $\mathcal{I}(M)\left(\operatorname{resp} . \mathcal{I}\left(M^{\prime}\right)\right)$ be the ideal generated by $r$ (resp. by $\left.r^{\prime}\right)$. Let $H:\left(\mathbb{C}^{N}, 0\right) \rightarrow\left(\mathbb{C}^{N}, 0\right)$ be a formal holomorphic map. We associate to the map $H$ another formal map $\mathcal{H}:\left(\mathbb{C}^{N} \times \mathbb{C}^{N}, 0\right) \rightarrow\left(\mathbb{C}^{N} \times \mathbb{C}^{N}, 0\right)$ defined by $\mathcal{H}(Z, \zeta)=(H(Z), \bar{H}(\zeta))$. We say that $H$ sends $M$ into $M^{\prime}$ if $\mathcal{I}\left(M^{\prime}\right) \subset \mathcal{H}_{*}(\mathcal{I}(M))$ and write $H(M) \subset M^{\prime}$. The formal map $H$ is $C R$-transversal if

$$
T_{0}^{1,0} M^{\prime}+d H\left(T_{0} \mathbb{C}^{N}\right)=T_{0} \mathbb{C}^{N},
$$

where $d H$ denotes the differential of $H$ (at 0 ).

If we choose normal coordinates $Z=(z, w) \in \mathbb{C}^{N}$ and $Z^{\prime}=\left(z^{\prime}, w^{\prime}\right) \in \mathbb{C}^{N}$ for $M$ and $M^{\prime}$ respectively, and if we write the map $H=(F, G) \in \mathbb{C}^{N-1} \times \mathbb{C}^{N}$, then the $H$ is CR-transversal if 
and only if $\partial G / \partial w(0) \not \equiv 0$ (see e.g. [5, 11]). We refer to the last component $G$ of $H$ in any system of normal coordinates as a transversal (or normal) component of $H$. When $G \equiv 0$, we say that $H$ is transversally flat. Following [5], we also say that $H$ is not totally degenerate if $z \mapsto \frac{\partial F}{\partial z}(z, 0)$ is of generic rank $N-1$. It is easy to see (and well-known) that the above conditions are independent of the choice of normal coordinates for $M, M^{\prime}$. Throughout the note, we will denote by Jac $H$ the usual Jacobian determinant of $H$.

\section{THE FINITE TYPE CASE}

2.1. A prolongation lemma. Our main transversality result in the finite case (Theorem 2.2 below) is based on the following result. In what follows, for every integer $k$, we denote by $J_{0}^{k}\left(\mathbb{C}^{n}, \mathbb{C}^{d}\right)$ the space of $k$-jets at 0 of holomorphic maps from $\mathbb{C}^{n}$ into $\mathbb{C}^{d}$.

Lemma 2.1. Let $A:\left(\mathbb{C}_{z}^{n} \times \mathbb{C}_{\chi}^{m},(0,0)\right) \rightarrow \mathbb{C}$ be a formal (holomorphic) power series, and assume that $\operatorname{ord}_{z} A(z, \chi)=k<\infty$. Then, for every positive integer $d$ and for each $\alpha \in \mathbb{N}^{n}$, there exists a universal linear map

$$
T_{\alpha}: J_{0}^{k+|\alpha|}\left(\mathbb{C}^{n}, \mathbb{C}^{d}\right) \rightarrow \mathbb{C}^{d}
$$

(over the quotient field of meromorphic powers series in $\chi$ ) such that for every formal power series mapping b: $\left(\mathbb{C}^{n} \times \mathbb{C}^{m}, 0\right) \rightarrow \mathbb{C}^{d}$ and every $v_{\beta}:\left(\mathbb{C}^{m}, 0\right) \rightarrow \mathbb{C}^{d}$ for $\beta \in \mathbb{N}^{n}$ satisfying

$$
\left.\left[\frac{\partial^{|\beta|}}{\partial z^{\beta}}(A(z, \chi) b(z, \chi))\right]\right|_{z=0}=v_{\beta}(\chi), \quad \text { for all } \beta \in \mathbb{N}^{n},
$$

then

$$
b_{z^{\alpha}}(0, \chi)=T_{\alpha}\left(\left(v_{\beta}(\chi)\right)_{|\beta| \leq|\alpha|+k}\right) .
$$

Proof. Note that the case $\operatorname{ord}_{z} A(z, \chi)=0$ is trivial (we can just divide by $A$ then), so we assume that $k>0$ for the proof.

We order the multiindeces in $\mathbb{N}^{n}$ by lexicographic ordering; that is, $\alpha<\beta$ if and only if $\alpha \neq \beta$ and for the least $j$ with $\alpha_{j}-\beta_{j} \neq 0$ this number is negative.

We first choose $\alpha^{0}$ to be the minimal multiindex $\alpha \in \mathbb{N}^{n}$ with $A_{z^{\alpha}}(0, \chi) \neq 0$, fix the integer $d$ and prove the lemma by induction on $\ell=|\alpha|$. For $\ell=0$, the result is obvious by using (2.2) for $\beta=\alpha^{0}$.

Now assume that we have constructed the maps $T_{\alpha}$ for $|\alpha|<\ell$ for some positive integer $\ell$. It is clear from the chain rule that the equations (2.2) for $|\beta|=\ell+k$ only involve the $b_{z \gamma}(0, \chi)$ for $|\gamma| \leq \ell$. We first replace, inside such equations, the terms $b_{z^{\gamma}}(0, \chi)$ by $T_{\gamma}\left(v_{\beta}(\chi):|\beta| \leq k+\ell-1\right)$ for all $\gamma$ of length strictly less than $\ell$. We now compute the coefficient of $b_{z \gamma}(0, \chi)$ for all $\gamma$ of length $\ell$. For every such multiindex, we consider the equation given by (2.2) for $\beta=\alpha^{0}+\gamma$.

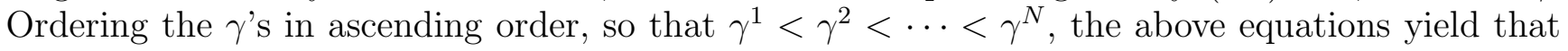
for every $j=1, \ldots, N$,

$$
\sum_{\substack{\gamma \geq \gamma^{j},|\gamma|=\ell}} A_{z^{\alpha^{0}+\gamma-\gamma^{j}}}(0, \chi) b_{z^{\gamma}}(0, \chi)=\rho_{j}\left(v_{\beta}(\chi):|\beta| \leq \ell+k\right)
$$

for some universal linear map $\rho_{j}: J_{0}^{k+\ell}\left(\mathbb{C}^{n}, \mathbb{C}^{d}\right) \rightarrow \mathbb{C}^{d}$ (over Frac $\mathbb{C}[[\chi]$ ) The obtained system is triangular in the unknowns $b_{z^{j}}(0, \chi), j=1, \ldots, N$ and can be solved. The proof of Lemma 2.1 is finished. 
2.2. Statement of the results. Our main result for finite type hypersurfaces is given by the following.

Theorem 2.2. Let $M, M^{\prime} \subset \mathbb{C}^{N}$ be formal real hypersurfaces with $M$ belonging to the class $\mathcal{C}$. Then every formal holomorphic map $H:\left(\mathbb{C}^{N}, 0\right) \rightarrow\left(\mathbb{C}^{N}, 0\right)$ sending $M$ into $M^{\prime}$ which is transversally nonflat is necessarily not totally degenerate, and hence CR-transversal.

As a first consequence of Theorem [2.2, we have the following result which provides a partial answer to a question posed in the paper [1] (see also Conjecture 2.7] below).

Corollary 2.3. Let $M, M^{\prime} \subset \mathbb{C}^{N}$ be as in Theorem 2.2 . Then every formal holomorphic map $H:\left(\mathbb{C}^{N}, 0\right) \rightarrow\left(\mathbb{C}^{N}, 0\right)$ sending $M$ into $M^{\prime}$ satisfying Jac $H \not \equiv 0$ is $C R$-transversal.

We also obtain the following transversality result for arbitrary mappings.

Corollary 2.4. Let $M, M^{\prime} \subset \mathbb{C}^{N}$ be formal real hypersurfaces. Assume that $M$ belongs to the class $\mathcal{C}$ and that $M^{\prime}$ does not contain any formal curve. Then any formal holomorphic map $H:\left(\mathbb{C}^{N}, 0\right) \rightarrow\left(\mathbb{C}^{N}, 0\right)$ sending $M$ into $M^{\prime}$ is either constant or CR-transversal.

Theorem [2.2 improves a transversality result due to Baouendi-Rothschild [5] established for essentially finite hypersurfaces. In fact, in this note, we will only show that the map $H$ in Theorem 2.2 is not totally degenerate, the CR-transversality of the map being then a consequence of the recent result of Ebenfelt-Rothschild [11, Theorem 4.1]. We should also point out that when $M$ is assumed to be essentially finite, it was shown in [5] that the map is in fact CR-transversal and finite. This need not be the case when $M$ is merely in the class $\mathcal{C}$ as shown by the following example.

Example 2.5. Let $\psi=\left(\psi_{1}, \ldots, \psi_{n}\right) \in(\mathbb{C}[[z]])^{n}$ be a formal map of generic rank $n$, not finite and vanishing at the origin. Consider the formal hypersurface $M_{\psi}:=\left\{(z, w) \in \mathbb{C}^{n} \times \mathbb{C}\right.$ : $\left.\operatorname{Im} w=\sum_{j=1}^{n}\left|\psi_{j}(z)\right|^{2}\right\}$ as well as the Heisenberg hypersurface $\mathbb{H}_{n}:=\left\{\left(z^{\prime}, w^{\prime}\right) \in \mathbb{C}^{n} \times \mathbb{C}: \operatorname{Im} w^{\prime}=\right.$ $\left.\sum_{j=1}^{n}\left|z_{j}^{\prime}\right|^{2}\right\}$. Then the hypersurface $M_{\psi} \in \mathcal{C}$ and the formal map $H$ given by $H(z, w)=(\psi(z), w)$ sends $M_{\psi}$ into $\mathbb{H}_{n}$, is CR-transversal, not totally degenerate and obviously not finite.

In fact, we have the following more precise statement.

Proposition 2.6. Let $M, M^{\prime} \subset \mathbb{C}^{N}$ be as in Theorem 2.2 and $H:\left(\mathbb{C}^{N}, 0\right) \rightarrow\left(\mathbb{C}^{N}, 0\right)$ be a formal holomorphic map sending $M$ into $M^{\prime}$. Then the following are equivalent:

(i) $H$ is CR transversal;

(ii) $H$ is not totally degenerate.

Note that Proposition 2.6] is not true in the more general situation when $M$ is holomorphically nondegenerate and of finite type (see for instance the example given in [11, p.26-27]). However, we believe that the following should be true:

Conjecture 2.7. Let $M, M^{\prime} \subset \mathbb{C}^{N}$ be formal real hypersurfaces with $M$ holomorphically nondegenerate and of finite type. Then the following are equivalent:

(i) $H$ is CR transversal;

(ii) $\operatorname{Jac} H \not \equiv 0$.

We support such a conjecture by proving the following result, which in particular shows that the implication (i) $\Rightarrow$ (ii) in Conjecture 2.7 is indeed true. 
Theorem 2.8. Let $M, M^{\prime} \subset \mathbb{C}^{N}$ be formal real hypersurfaces with $M$ holomorphically nondegenerate. Then every formal holomorphic map $H:\left(\mathbb{C}^{N}, 0\right) \rightarrow\left(\mathbb{C}^{N}, 0\right)$ sending $M$ into $M^{\prime}$ which is transversally nonflat satisfies Jac $H \not \equiv 0$.

The proof of Theorem 2.8 will be obtained by following the lines of the proof of Theorem 2.2 . Note however that, contrarily to Theorem 2.2. the map $H$ in Theorem 2.8 need not be CRtransversal as the basic example $M=\left\{(z, w) \in \mathbb{C}^{2}: \operatorname{Im} w=|z w|^{2}\right\}, M^{\prime}=\mathbb{H}_{1}$ and $H(z, w)=$ $(z, z w)$, shows.

\subsection{Proofs of the results.}

Proof of Theorem 2.2. Choose normal coordinates $Z=(z, w)$ for $M$ so that $M$ is given by (1.1) and similarly for $M^{\prime}$ (we just add a "prime" to the corresponding objects for the target hypersurface) and write $H=(F, G)=\left(F^{1}, \ldots, F^{N-1}, G\right)$ as in $\S$ 1. Assume, by contradiction, that $H$ is totally degenerate. We will show that this forces the transversal component $G$ to vanish. Since $H$ sends $M$ into $M^{\prime}$, we have the power series identity

$$
G(z, Q(z, \chi, 0))=Q^{\prime}(F(z, Q(z, \chi, 0)), \bar{F}(\chi, 0), 0)
$$

that we differentiate with respect to $\chi$ to obtain

$$
\begin{aligned}
\left(G_{w}(z, Q(z, \chi, 0))-Q_{z^{\prime}}^{\prime}(F(z, Q(z, \chi, 0)), \bar{F}(\chi, 0), 0) \cdot F_{w}(z, Q(z, \chi, 0)) Q_{\chi}(z, \chi, 0)\right. & \\
& =Q_{\chi^{\prime}}^{\prime}(F(z, Q(z, \chi, 0)), \bar{F}(\chi, 0), 0) \cdot \frac{\partial \bar{F}}{\partial \chi}(\chi, 0) .
\end{aligned}
$$

Taking derivatives (of arbitrary order) with respect to $z$ and evaluating at 0 we see that the right hand side of (2.5) is always contained in the (C-linear) span of the $\bar{F}_{\chi}^{j}(\chi, 0), j=1, \ldots, N-1$; this space has by assumption dimension less than $N-1$ (strictly). If the coefficient of $Q_{\chi}(z, \chi, 0)$ on the left hand side of (2.5) is not zero, an application of Lemma 2.1 (with $b(z, \chi)=Q_{\chi}(z, \chi, 0)$ ) yields that for every $\alpha \in \mathbb{N}^{N-1}, Q_{z^{\alpha}, \chi}(0, \chi, 0)$ is contained in the (Frac $\mathbb{C}[[\chi]]$-linear) span of the $\bar{F}_{\chi}^{j}(\chi, 0), j=1, \ldots, N-1$, a contradiction, since $M \in \mathcal{C}$ means that the dimension of the space spanned (over Frac $\mathbb{C}[[\chi])$ ) by the $Q_{z^{\alpha}, \chi}(0, \chi, 0), \alpha \in \mathbb{N}^{N-1}$, is $N-1$.

Thus, we conclude that

$$
G_{w}(z, Q(z, \chi, 0))-Q_{z^{\prime}}^{\prime}(F(z, Q(z, \chi, 0)), \bar{F}(\chi, 0), 0) \cdot F_{w}(z, Q(z, \chi, 0))=0 .
$$

Setting $\chi=0$ in this equation we have that $G_{w}(z, 0)=0$. We now construct by induction for each positive integer $k$ formal power series $c^{k}\left(z, w, \chi^{\prime}\right)$ satisfying

$$
\begin{aligned}
c^{k}(z, w, 0) & =0, \\
G_{w^{k}}(z, Q(z, \chi, 0))-c^{k}(z, Q(z, \chi, 0), \bar{f}(\chi, 0)) & =0 .
\end{aligned}
$$

In view of (2.6), we have already constructed $c^{1}$. For every integer $\ell \geq 2$, if $c^{1}, \ldots, c^{\ell-1}$ have been constructed, we then differentiate the equation (2.8) for $k=\ell-1$ with respect to $\chi$ and we obtain

$$
\begin{aligned}
\left(G_{w^{\ell}}(z, Q(z, \chi, 0))-c_{w}^{\ell-1}(z, Q(z, \chi, 0), \bar{F}(\chi, 0))\right) & Q_{\chi}(z, \chi, 0) \\
& =c_{\chi^{\prime}}^{\ell-1}(z, Q(z, \chi, 0), \bar{F}(\chi, 0)) \cdot \frac{\partial \bar{F}}{\partial \chi}(\chi, 0) .
\end{aligned}
$$


The same argument as in the case $\ell=1$ above lets us conclude that the coefficient of $Q_{\chi}(z, \chi, 0)$ on the left hand side of (2.9) vanishes. Thus, we can choose

$$
c^{\ell}\left(z, w, \chi^{\prime}\right):=c_{w}^{\ell-1}\left(z, w, \chi^{\prime}\right),
$$

which satisfies (2.7) and (2.8) for $k=\ell$. We may now conclude that $G_{w^{\ell}}(z, 0)=0$ for all $\ell$, so that the transversal component of $H$ is flat. The proof of the theorem is complete.

For the proof of Corollary 2.4, we need the following result contained in the proof of [2, Proposition 7.1].

Lemma 2.9. Let $M, M^{\prime} \subset \mathbb{C}^{N}$ be formal real hypersurfaces. Assume that $M$ is holomorphically nondegenerate and that $M^{\prime}$ does not contain any formal curve. Then every formal holomorphic map sending $M$ into $M^{\prime}$ that is transversally flat is necessarily constant.

Proof of Corollary 2.4. The corollary is obtained by combining Theorem 2.2 and Lemma 2.9] and noticing that if $M \in \mathcal{C}$ then $M$ is holomorphically nondegenerate.

Proof of Proposition 2.6. The implication (i) $\Rightarrow($ ii) is proved in [17, Corollary 4.2] while the implication (i) $\Rightarrow$ (ii) (with the weaker assumption that $M$ is of finite type instead of being in the class $\mathcal{C})$ is the content of [11, Theorem 4.1].

Proof of Theorem 2.8. We keep the notation of the proof of Theorem 2.2 and present a completely analogous argument. We also write $\zeta=(\chi, \tau) \in \mathbb{C}^{N-1} \times \mathbb{C}$ and $H=(F, G)=\left(H^{1}, \ldots, H^{N}\right)$. Here again assume, by contradiction, that Jac $H \equiv 0$. We will show that this forces the transversal component $G$ to vanish. Since $H$ sends $M$ into $M^{\prime}$, we have the formal power series relation

$$
G(z, Q(z, \zeta))=Q^{\prime}(F(z, Q(z, \zeta)), \bar{H}(\zeta)) .
$$

We differentiate (2.10) with respect to $\zeta$ to get

$$
\begin{aligned}
\left(G_{w}(z, Q(z, \zeta))-Q_{z^{\prime}}^{\prime}(F(z, Q(z, \zeta)), \bar{H}(\zeta)) \cdot F_{w}(z, Q(z, \zeta)) Q_{\zeta}(z, \zeta)\right. & \\
& =Q_{\zeta^{\prime}}^{\prime}(F(z, Q(z, \zeta)), \bar{H}(\zeta)) \cdot \frac{\partial \bar{H}}{\partial \zeta}(\zeta)
\end{aligned}
$$

Taking derivatives (of arbitrary order) with respect to $z$ and evaluating at 0 we see that the right hand side of (2.11) is always contained in the (C-linear) span of the $\bar{H}_{\zeta}^{j}(\zeta), j=1, \ldots, N$; this space has by assumption dimension less than $N$ (strictly). If the coefficient of $Q_{\zeta}(z, \zeta)$ on the left hand side of (2.11) is not zero, another application of Lemma 2.1 yields that for every $\alpha \in \mathbb{N}^{N-1}$, $Q_{z^{\alpha}, \zeta}(0, \zeta)$ is contained in the (Frac $\mathbb{C}[[\zeta]]$-linear) span of the $\bar{H}_{\zeta}^{j}(\zeta), j=1, \ldots, N$, a contradiction, since $M$ being holomorphically nondegenerate means that the dimension of the space spanned (over Frac $\mathbb{C}[[\zeta]]$ ) by the $Q_{z^{\alpha}, \zeta}(0, \zeta), \alpha \in \mathbb{N}^{N-1}$, is $N$ (see e.g. [1, 21]).

We therefore conclude that

$$
G_{w}(z, Q(z, \zeta))-Q_{z^{\prime}}^{\prime}(F(z, Q(z, \zeta)), \bar{H}(\zeta)) \cdot F_{w}(z, Q(z, \zeta))=0 .
$$

Setting $\zeta=0$ in (2.12) we get that $G_{w}(z, 0)=0$. As in the proof of Theorem 2.2, we define by induction for each positive integer $k$ formal power series $S^{k}\left(z, w, \zeta^{\prime}\right)$ satisfying

$$
\begin{aligned}
S^{k}(z, w, 0) & =0, \\
G_{w^{k}}(z, Q(z, \zeta))-S^{k}(z, Q(z, \zeta), \bar{H}(\zeta)) & =0 .
\end{aligned}
$$


We have already constructed $S^{1}$ above. For every integer $\ell \geq 2$, if $S^{1}, \ldots, S^{\ell-1}$ have been defined, we then differentiate the equation (2.14) for $k=\ell-1$ with respect to $\zeta$ and obtain

$$
\left(G_{w^{\ell}}(z, Q(z, \zeta))-S_{w}^{\ell-1}(z, Q(z, \zeta), \bar{H}(\zeta))\right) Q_{\zeta}(z, \zeta)=S_{\zeta^{\prime}}^{\ell-1}(z, Q(z, \zeta), \bar{H}(\zeta)) \cdot \frac{\partial \bar{H}}{\partial \zeta}(\zeta)
$$

The same argument as in the case $\ell=1$ above lets us conclude that the coefficient of $Q_{\zeta}(z, \zeta)$ on the left hand side of (2.15) vanishes. Thus, we can choose

$$
S^{\ell}\left(z, w, \zeta^{\prime}\right):=S_{w}^{\ell-1}\left(z, w, \zeta^{\prime}\right),
$$

which satisfies (2.13) and (2.14) for $k=\ell$. All this implies that $G_{w^{\ell}}(z, 0)=0$ for all $\ell$, so that the transversal component of $H$ is flat. The proof of the theorem is finished.

\section{THE INFINITE TYPE CASE}

In this section, we will derive some analogues of the transversality result due to Baouendi and Rothschild [5] for infinite type hypersurfaces. We say that a formal real hypersurface $M \subset \mathbb{C}^{N}$ given in normal coordinates $(z, w) \in \mathbb{C}^{N}$ is of $m$-infinite type, where $m \in \mathbb{Z}_{+}$, if $M$ is given by a (complexified) defining equation of the form

$$
w=Q(z, \chi, \tau), \text { where } Q(z, \chi, \tau)=\tau+\tau^{m} \widetilde{Q}(z, \chi, \tau),
$$

with $\widetilde{Q}(z, 0, \tau)=\widetilde{Q}(0, \chi, \tau)=0$ and

$$
\widetilde{Q}(z, \chi, 0) \not \equiv 0 \text {. }
$$

(As customary, we use a similar notation for any other formal real hypersurface $M^{\prime}$ by just adding a "prime".) This above condition was introduced by Meylan [19, who also observed that such a condition together with the integer $m$ are invariantly attached to $M$ (i.e. independent of the chosen normal coordinates); the notion of "transversal order" that we define next is also due to Meylan, who established its basic properties.

3.1. The transversal order of a map. Let $M, M^{\prime} \subset \mathbb{C}^{N}$ be two formal real hypersurfaces (given in normal coordinates) that we assume to be of $m$-infinite and $m^{\prime}$-infinite type respectively, $m, m^{\prime} \geq 1$. Given a transversally nonflat formal holomorphic map $H:\left(\mathbb{C}^{N}, 0\right) \rightarrow\left(\mathbb{C}^{N}, 0\right)$ sending $M$ into $M^{\prime}$ written $H=(F, G)$ as in \$1 we define the transversal order of $H$ and denote it by trord $H$ to be the integer $k$ such that $G(z, w)=w^{k} \widetilde{G}(z, w)$ with $\widetilde{G}(z, 0) \not \equiv 0$. If $H$ is transversally flat, we just naturally set trord $H=\infty$. We note that in any case we always have trord $H \geq 1$ and that the notion of transversal order is invariant under changes of coordinates preserving normal coordinates in the source and in the target space [19, Lemma 2.1].

The first lemma we state asserts that in the above situation we necessarily have $\widetilde{G}(0) \neq 0$. It is also contained in [19] but for the reader's convenience we give a short proof.

Lemma 3.1. Let $M, M^{\prime}$ be as above and $H:\left(\mathbb{C}^{N}, 0\right) \rightarrow\left(\mathbb{C}^{N}, 0\right)$ be a formal holomorphic map sending $M$ into $M^{\prime}$. If $H$ is not transversally flat, that is, trord $H=k<\infty$, then $G_{w^{k}}(z, 0)=$ $G_{w^{k}}(0) \in \mathbb{R} \backslash\{0\}$.

Proof. We start with the equation

$$
G(z, Q(z, \chi, \tau))=Q^{\prime}(F(z, Q(z, \chi, \tau)), \bar{F}(\chi, \tau), \bar{G}(\chi, \tau)),
$$


in which we set $\chi=0, \tau=w$. This gives

$$
G(z, w)=\bar{G}(0, w)+\bar{G}(0, w)^{m^{\prime}} \widetilde{Q}^{\prime}(F(z, w), \bar{F}(0, w), \bar{G}(0, w)) .
$$

The last equation clearly implies that $G(0, w) \neq 0$; so let $s$ be the smallest integer such that $G_{w^{s}}(0) \neq 0$. From the definition of $k$ and $s$, we immediately get that $s \geq k$. Furthermore we notice that the order (in $w$ ) of left-hand side of (3.3) is exactly $k$, whereas it is at least $s$ for the right-hand side of (3.3) which shows that $k \geq s$ and therefore $k=s$. Now comparing the coefficients of $w^{k}$ on both sides of the equation we get $G_{w^{k}}(z, 0)=\overline{G_{w^{k}}}(0)$, which completes the proof of the lemma.

Our goal in this section is to bound the transversal order of a map in terms of $m$ and $m^{\prime}$. The bound is provided in our next proposition.

Proposition 3.2. Let $M, M^{\prime}$ be as above and $H:\left(\mathbb{C}^{N}, 0\right) \rightarrow\left(\mathbb{C}^{N}, 0\right)$ be a formal holomorphic map sending $M$ into $M^{\prime}$. If $H$ is transversally nonflat, then

$$
\left(m^{\prime}-1\right) \text { trord } H \leq m-1 \text {. }
$$

We will give examples illustrating this bound with specific maps in Example 3.5. Let us remark that if $m^{\prime}=1$, there are examples to show that no bound on the transversal order can be obtained in this case, see Example 3.6 below. Let us also add that if, in Proposition 3.2, the map $H$ is assumed to be furthermore not totally degenerate, Meylan proved that the inequality given by (3.4) is in fact an equality (see [19, Proposition 2.2]).

Proof. We again start with (3.2), set trord $H=k$, and rewrite this equation in the following way:

$$
\begin{aligned}
\left(\tau+\tau^{m} \tilde{Q}(z, \chi, \tau)\right)^{k} \widetilde{G}(z, Q(z, \chi, \tau)) & = \\
\tau^{k} \widetilde{\widetilde{G}}(\chi, \tau) & +\tau^{k m^{\prime}} \overline{\widetilde{G}}(\chi, \tau)^{m^{\prime}} \widetilde{Q}^{\prime}(F(z, Q(z, \chi, \tau)), \bar{F}(\chi, \tau), \bar{G}(\chi, \tau)) .
\end{aligned}
$$

On the left hand side of this equation, we claim that there is a term which has order $k+m-1$ in $\tau$ whose coefficient depends on $z$ (and $\chi$ ). Indeed, let us assume that

$$
\tilde{Q}(z, \chi, 0)=\sum_{(a, b) \in\left(\mathbb{Z}_{+}\right)^{2}} \tilde{Q}_{a, b}(z, \chi, 0), \quad \text { where } \tilde{Q}_{a, b}(\lambda z, \mu \chi, 0)=\lambda^{a} \mu^{b} \tilde{Q}_{a, b}(z, \chi, 0), \quad(\lambda, \mu) \in \mathbb{C}^{2},
$$

and let $\left(a_{0}, b_{0}\right)$ be minimal under the condition that $\tilde{Q}_{a, b}(z, \chi, 0)=0$ for $a+b<a_{0}+b_{0}$ and $a<a_{0}$. We expand the series on the left hand side of (3.5) in terms $T_{a, b, c}(z, \chi, \tau)$, which are homogeneous in $z, \chi$, and $\tau$ i.e. $T_{a, b, c}(\lambda z, \mu \chi, \delta \tau)=\lambda^{a} \mu^{b} \delta^{c} T_{a, b, c}(z, \chi, \tau)$ for $\lambda, \mu$ as above and $\delta \in \mathbb{C}$. We claim that $T_{a_{0}, b_{0}, k+m-1}(z, \chi, \tau)=k \widetilde{G}(0) \tau^{k+m-1} \tilde{Q}_{a_{0}, b_{0}}(z, \chi, 0) \neq 0$. First note that modulo terms $T_{a, b, c}$ with $c>k+m-1$, we can rewrite the series as $\left(\tau+\tau^{m} \tilde{Q}(z, \chi, 0)\right)^{k} \widetilde{G}(z, \tau)$. We now pick the terms which are homogeneous of order $a_{0}$ in $z$ and $b_{0}$ in $\chi$ and see that our claim holds.

Now, the lowest order in $\tau$ of a term of the series on the right hand side of (3.5) which contains a coefficient depending on $z$ is at least $k m^{\prime}$. Thus, $k m^{\prime} \leq k+m-1$, which is the inequality we wanted to prove.

Given a formal real hypersurface $M \subset \mathbb{C}^{N}, M$ is of infinite type if and only if there is a formal complex hypersurface $E$, which we refer to as the "exceptional" complex hypersurface, which is contained in $M$. (In normal coordinates, this is necessarily given by $E=\{w=0\}$.) Note that if $M$ is such a hypersurface, a necessary condition for a formal holomorphic self-map of $M$ to be CR-transversal is to be transversally non-flat i.e. $H\left(\mathbb{C}^{N}\right) \not \subset E$. As a direct application 
of Proposition 3.2 we obtain that the converse of this statement does hold for $m$-infinite type hypersurfaces provided that $m \geq 2$. In addition, Example 3.6 below shows that the corresponding result does not hold for 1-infinite type hypersurfaces.

Corollary 3.3. Let $M \subset \mathbb{C}^{N}$ be a formal real hypersurface of $m$-infinite type with $m \geq 2$, and denote by $E$ the exceptional complex hypersurface contained in $M$. Then for every formal holomorphic map $H:\left(\mathbb{C}^{N}, 0\right) \rightarrow\left(\mathbb{C}^{N}, 0\right)$ sending $M$ into itself, either $H$ is CR-transversal or $H\left(\mathbb{C}^{N}\right) \subset E$.

Another application of Proposition 3.2 is given by the following generalization of Corollary 3.3 .

Corollary 3.4. Let $M, M^{\prime} \subset \mathbb{C}^{N}$ be formal real hypersurfaces of $m$-infinite and $m^{\prime}$-infinite type respectively, and denote by $E^{\prime}$ the exceptional complex hypersurface contained in $M^{\prime}$. Assume that $1<m^{\prime} \leq m$ and that $m<2 m^{\prime}-1$. Then for every formal holomorphic map $H:\left(\mathbb{C}^{N}, 0\right) \rightarrow\left(\mathbb{C}^{N}, 0\right)$ sending $M$ into $M^{\prime}$, either $H$ is CR-transversal or $H\left(\mathbb{C}^{N}\right) \subset E^{\prime}$.

We will now discuss the examples we already pointed out. Our first example provides explicit maps between certain infinite type hypersurfaces, where we can compute the infinite type and the transversal order of the maps explicitly.

Example 3.5. Let us note that (for different reasons) certain special cases of this example have been considered by Kowalski 15 and Zaitsev 22 .

Consider the Heisenberg hypersurface $\mathbb{H}_{1} \subset \mathbb{C}_{(z, w)}^{2}$ as given in Example 2.5. We let

$$
H_{b, c}(z, w)=\left(\sqrt{c} z w^{b}, w^{c}\right), \quad b, c \in \mathbb{Z}_{+},
$$

be a weighted analytic blowup of $\mathbb{C}^{2}$; for reasons which will become apparent below, we concentrate on blowups where $2 b>c$. The preimage of $\mathbb{H}_{1}$ under this weighted blowup is given by

$$
\operatorname{Im} w^{c}=c|z|^{2}|w|^{2 b}
$$

setting $w=s+i t$, this equation turns into

$$
\sum_{k=0}^{[(c-1) / 2]}\left(\begin{array}{c}
c \\
2 k+1
\end{array}\right)(-1)^{k} s^{c-2 k-1} t^{2 k+1}=c|z|^{2} \sum_{k=0}^{b}\left(\begin{array}{l}
b \\
k
\end{array}\right) s^{2 b-2 k} t^{2 k},
$$

where $[\cdot]$ denotes the integer part. We set $s^{d} u=t$, where $d=2 b-c+1$; after division by $s^{2 b}$ the equation above now becomes

$$
\sum_{k=0}^{[(c-1) / 2]}\left(\begin{array}{c}
c \\
2 k+1
\end{array}\right)(-1)^{k} s^{2 k(d-1)} u^{2 k+1}=c|z|^{2} \sum_{k=0}^{b}\left(\begin{array}{l}
b \\
k
\end{array}\right) s^{2 k(d-1)} u^{2 k}
$$

By the Implicit Function Theorem, this equation has a unique analytic solution $u=\Theta_{b, c}\left(|z|^{2}, s\right)$ which satisfies $\Theta_{b, c}\left(|z|^{2}, 0\right)=|z|^{2} \neq 0$ and $\Theta_{b, c}(0, s)=0$ (here we use that $2 b>c$ ). Thus, the preimage of $\mathbb{H}_{1}$ under the blowup $H_{b, c}$ contains a germ of a real-analytic hypersurface $M_{b, c}$, of $d$-infinite type given by the equation

$$
t=s^{d} \Theta_{b, c}\left(|z|^{2}, s\right) .
$$

From this construction, we obtain examples of maps between hypersurfaces of infinite type by noticing that $H_{b, c} \circ H_{\tilde{b}, \tilde{c}}=H_{\tilde{b}+b \tilde{c}, c \tilde{c}}$ with $2 \tilde{b}>\tilde{c}, \tilde{b}, \tilde{c} \in \mathbb{Z}_{+}$; we claim that $H_{\tilde{b}, \tilde{c}}$ is a map of transversal 
order $\tilde{c}$ from the $m$-infinite type hypersurface $M_{\tilde{b}+b \tilde{c}, \tilde{c} \tilde{c}}$ to the $m^{\prime}$-infinite type hypersurface $M_{b, c}$ where $m=2(\tilde{b}+b \tilde{c})-c \tilde{c}+1$ and $m^{\prime}=2 b-c+1$; for this map, the bound (3.4) becomes

$$
\operatorname{trord} H_{\tilde{b}, \tilde{c}}=\tilde{c} \leq \frac{m-1}{m^{\prime}-1}=\tilde{c}+\frac{2 \tilde{b}}{2 b-c} .
$$

Let us establish our claim above i.e. that $H_{\tilde{b}, \tilde{c}}$ actually sends $M_{\tilde{b}+b \tilde{c}, c \tilde{c}}$ into $M_{b, c}$. We rewrite the defining equation of $M_{b, c}$ in complex form and replace the variables by $(\zeta, \eta)$; then, $M_{b, c} \subset \mathbb{C}_{\zeta, \eta}^{2}$ is given by

$$
\eta=\bar{\eta}+\bar{\eta}^{m^{\prime}} \Phi\left(|\zeta|^{2}, \bar{\eta}\right)
$$

where now $\Phi\left(|\zeta|^{2}, 0\right)=2 i|\zeta|^{2}$ and $\Phi(0, s)=0$. To prove our claim, we will show that there is exactly one germ of a real-analytic hypersurface in $\mathbb{C}_{z, w}^{2}$ of the form $t=s^{m} \varphi\left(|z|^{2}, s\right), w=s+i t$, with $\varphi\left(|z|^{2}, 0\right)=|z|^{2}$ in the preimage of $M_{b, c}$ under the blowup $H_{\tilde{b}, \tilde{c}}$ with $m=\left(m^{\prime}-1\right) \tilde{c}+2 \tilde{b}+1$; since the preimage of $\mathbb{H}$ under the composition $H_{b, c} \circ H_{\tilde{b}, \tilde{c}}$ is another such, the real-analytic hypersurface constructed in this way in the preimage of $M_{b, c}$ under $H_{\tilde{b}, \tilde{c}}$ agrees with $M_{\tilde{b}+b \tilde{c}, c \tilde{c}}$.

As before, we substitute $\eta=w^{\tilde{c}}$, and $\zeta=\sqrt{\tilde{c}} z w^{\tilde{b}}$. The equation of $H_{\tilde{b}, \tilde{c}}^{-1}\left(M_{b, c}\right)$ turns into

$$
2 i \sum_{j}\left(\begin{array}{c}
\tilde{c} \\
2 j+1
\end{array}\right)(-1)^{j} s^{\tilde{c}-(2 j+1)} t^{2 j+1}=(s-i t)^{\tilde{c} m^{\prime}} \Phi\left(\tilde{c}|z|^{2}\left(s^{2}+t^{2}\right)^{\tilde{b}},(s-i t)^{\tilde{c}}\right) .
$$

We now proceed as above and substitute $t=\tilde{t} s^{m}$, where $m=\left(m^{\prime}-1\right) \tilde{c}+1+2 \tilde{b}$. Writing $\Phi$ as a Taylor series $\Phi\left(|\zeta|^{2}, \bar{\eta}\right)=\sum_{k \geq 1, l \geq 0} \Phi_{k, l}|\zeta|^{2 k} \bar{\eta}^{l}$

$$
\begin{aligned}
\Phi\left(\tilde{c}|z|^{2}\left(s^{2}+t^{2}\right)^{\tilde{b}},(s-i t)^{\tilde{c}}\right) & =\sum_{k \geq 1, l \geq 0} \Phi_{k, l}\left(s^{2}+\tilde{t}^{2} s^{2 m}\right)^{\tilde{b} k} \tilde{c}^{k}|z|^{2 k}\left(s-i \tilde{t} s^{m}\right)^{\tilde{c} l} \\
& =s^{2 \tilde{b}} \sum_{k \geq 1, l \geq 0} \Phi_{k, l} s^{2 \tilde{b}(k-1)+\tilde{c} l}\left(1+\tilde{t}^{2} s^{2 m-2}\right)^{\tilde{b} k} \tilde{c}^{k}|z|^{2 k}\left(1-i \tilde{t} s^{m-1}\right)^{\tilde{c} l} \\
& =s^{2 \tilde{b}} 2 i \tilde{c}|z|^{2}+O\left(s^{2 \tilde{b}+1}\right)
\end{aligned}
$$

we see that the right hand side of (3.6) is divisible by $s^{m^{\prime} \tilde{c}+2 \tilde{b}}$ (as is the left hand side), and after division, our equation becomes

$$
2 i \tilde{t}=2 i|z|^{2}+O(s)
$$

Here, we have again tacitly used the fact that since $2 b>c$ and $m^{\prime}>1, m>1$, and the Implicit Function Theorem guarantees a unique solution $\tilde{t}=\varphi\left(|z|^{2}, s\right)$ with $\varphi\left(|z|^{2}, 0\right)=|z|^{2}$ of this equation as claimed.

Example 3.6. For each positive integer $k$, the map $T_{k}: \mathbb{C}^{2} \rightarrow \mathbb{C}^{2}$ given by $T_{k}(z, w)=\left(z, w^{k}\right)$ maps the 1-infinite type hypersurface $M_{k}$ given by

$$
w=\bar{w} e^{\frac{i|z|^{2}}{k}}
$$

into the 1-infinite type hypersurface $M$ given by

$$
w=\bar{w} e^{i|z|^{2}} .
$$

Thus, the maps

$$
H_{k}(z, w)=\left(\sqrt{k} z, w^{k}\right)
$$


are examples of holomorphic self-maps sending the 1-infinite type hypersurface $M$ into itself having arbitrary transversal order.

3.2. On being an automorphism in the infinite type case. We conclude this note by proving an analogue in the infinite type case of a criterion (established in [17]) for a map to be an automorphism. We know from Corollary 3.3 that if $M$ a formal real hypersurface that is of $m$-infinite type with $m \geq 2$, then every formal holomorphic self-map $H$ of $M$ is either CR-transversal or transversally flat. We now put an additional nondegeneracy assumption on $M$ that will force any formal CR-transversal self-map of $M$ to be an automorphism (this will also be true in the 1-infinite type case, where in view of Example 3.6. the transversality result does not hold in general, see Theorem 3.10). For this, we say that $M$ belongs to the class $\mathcal{C}_{m}$ if $M$ is of $m$-infinite type and is given in normal coordinates as follows

$$
w=Q(z, \chi, \tau), \quad Q(z, \chi, \tau)=\tau+\tau^{m} \widetilde{Q}(z, \chi, \tau),
$$

with $\widetilde{Q}(z, \chi, 0)=\sum_{\alpha \in \mathbb{N}^{N-1}} \widetilde{Q}_{\alpha}(\chi) z^{\alpha}$, and with the formal map

$$
\chi \mapsto\left(\widetilde{Q}_{\alpha}(\chi)\right)_{|\alpha| \leq k}
$$

of generic rank $N-1$ for large enough $k$. We leave it to the reader to check that this condition is independent of the choice of normal coordinates.

Our goal in this section is to establish the following theorem.

Theorem 3.7. Let $M \subset \mathbb{C}^{N}$ be a formal real hypersurface that belongs to the class $\mathcal{C}_{m}$ for some $m \geq 2$ and denote by $E$ the exceptional complex hypersurface contained in $M$. Then for every formal holomorphic map $H:\left(\mathbb{C}^{N}, 0\right) \rightarrow\left(\mathbb{C}^{N}, 0\right)$ sending $M$ into itself, one of the following two conditions holds:

(i) $H\left(\mathbb{C}^{N}\right) \subset E$;

(ii) $H$ is an automorphism.

Note here again that Example [3.6] shows that Theorem 3.7 does not hold in the 1-infinite type case.

We start with the following lemma that can be found in [16, Lemma 3.8] for which we provide a different and simpler proof.

Lemma 3.8. Let $M \subset \mathbb{C}^{N}$ be a formal real hypersurface of $m$-infinite type with $m \geq 1$ and given in normal coordinates as above. Then for every formal CR-transversal holomorphic map $H:\left(\mathbb{C}^{N}, 0\right) \rightarrow\left(\mathbb{C}^{N}, 0\right)$ sending $M$ into itself, $H=(F, G)$, we have

$$
\widetilde{Q}(z, \chi, 0)=G_{w}(0)^{m-1} \widetilde{Q}(F(z, 0), \bar{F}(\chi, 0), 0) .
$$

Proof. For the proof, we start (as usual) with the identity

$$
G(z, Q(z, \chi, \tau))=Q(F(z, Q(z, \chi, \tau)), \bar{F}(\chi, \tau), \bar{G}(\chi, \tau)),
$$

and we write $G(z, w)=w \widetilde{G}(z, w)$ with $\widetilde{G}(z, 0)=G_{w}(0) \in \mathbb{R} \backslash\{0\}$ in view of Lemma 3.1. We may rewrite (3.9) as follows

$$
\begin{aligned}
& \left(\tau+\tau^{m} \widetilde{Q}(z, \chi, \tau)\right) \widetilde{G}(z, Q(z, \chi, \tau))= \\
& \bar{G}(\chi, \tau)+(\bar{G}(\chi, \tau))^{m} \widetilde{Q}(F(z, Q(z, \chi, \tau)), \bar{F}(\chi, \tau), \bar{G}(\chi, \tau)) .
\end{aligned}
$$


We look at the terms of order $m$ in $\tau$ in the above equation. On the left side, such a term is given by the quantity

$$
\widetilde{G}_{w^{m-1}}(z, 0)+\widetilde{Q}(z, \chi, 0) G_{w}(0),
$$

while on the right hand side, it is given by

$$
\bar{G}_{\tau^{m}}(\chi, 0)+\left(\bar{G}_{\tau}(0)\right)^{m} \widetilde{Q}(F(z, 0), \bar{F}(\chi, 0), 0) .
$$

Since $(\underline{3.11})=(3.12)$, we first get, after setting consecutively $z=0$ and $\chi=0$ in the obtained identity that $\widetilde{G}_{w^{m-1}}(z, 0)$ and $\bar{G}_{\tau^{m}}(\chi, 0)$ are equal and constant. This leads to the desired identity. The proof of the lemma is complete.

To prove Theorem 3.7. we need the following general fact about power series.

Lemma 3.9. Let $A:\left(\mathbb{C}_{z}^{n} \times \mathbb{C}_{\chi}^{n},(0,0)\right) \rightarrow \mathbb{C}$ be a formal power series such that for $k$ large enough the map

$$
\chi \mapsto\left(A_{z^{\alpha}}(0, \chi)\right)_{|\alpha| \leq k}
$$

is of generic rank $n$. If for some formal map $B:\left(\mathbb{C}^{n}, 0\right) \rightarrow\left(\mathbb{C}^{n}, 0\right)$ there is a nonzero constant $r$ such that

$$
A(z, \chi)=r A(B(z), \bar{B}(\chi)),
$$

then necessarily $B$ is a formal automorphism of $\mathbb{C}^{n}$.

Proof. From the equation $A(z, \chi)=r A(B(z), \bar{B}(\chi))$ we obtain for each $\alpha \in \mathbb{N}^{n}$ a polynomial map $\Phi_{\alpha}$ such that $A_{z^{\alpha}}(0, \chi)=\Phi_{\alpha}\left(\left(A_{z^{\beta}}(0, \bar{B}(\chi))\right)_{|\beta| \leq|\alpha|}\right)$. We choose $k$ large enough such that

$$
\chi \mapsto\left(A_{z^{\alpha}}(0, \chi)\right)_{|\alpha| \leq k}
$$

is of generic rank $n$ and also choose $n$ multiindeces $\alpha^{(1)}, \ldots, \alpha^{(n)}$ such that the order $\nu$ of the determinant of the matrix

$$
\left(\begin{array}{ccc}
\frac{\partial A_{z^{\alpha}}(1)}{\partial \chi_{1}}(0, \chi) & \ldots & \frac{\partial A_{z^{\alpha}}(1)}{\partial \chi_{n}}(0, \chi) \\
\vdots & & \vdots \\
\frac{\partial A_{z^{\alpha}}(n)}{\partial \chi_{1}}(0, \chi) & \ldots & \frac{\partial A_{z^{\alpha}}(n)}{\partial \chi_{n}}(0, \chi)
\end{array}\right)
$$

is minimal among any such choices of $n$ multiindeces. Differentiating the $n$ equations

$$
A_{z^{\alpha}(j)}(0, \chi)=\Phi_{\alpha^{(j)}}\left(\left(A_{z^{\beta}}(0, \bar{B}(\chi))_{|\beta| \leq\left|\alpha^{(j)}\right|}\right), \quad j=1, \ldots, n,\right.
$$

with respect to $\chi$ and using the Cauchy-Binet formula yields the inequality $\nu \geq \nu+\operatorname{ord} \operatorname{det} \bar{B}_{\chi}(\chi)$. Consequently, ord det $\bar{B}_{\chi}(\chi)=0$ i.e. $\operatorname{det} B_{z}(0) \neq 0$.

Combining the last two lemmas, we obtain the following result whose analogous statement is contained in [17] for hypersurfaces belonging to the class $\mathcal{C}$.

Theorem 3.10. Let $M \subset \mathbb{C}^{N}$ be a formal real hypersurface that belongs to the class $\mathcal{C}_{m}$ for some $m \geq 1$. Then every formal CR-transversal holomorphic map $H:\left(\mathbb{C}^{N}, 0\right) \rightarrow\left(\mathbb{C}^{N}, 0\right)$ sending $M$ into itself is an automorphism.

Proof of Theorem 3.7. The theorem is just obtained as the conjunction of Theorem 3.10 and Corollary 3.3 . 


\section{REFERENCES}

[1] M. S. Baouendi, P. Ebenfelt, and L. P. Rothschild. Real submanifolds in complex space and their mappings. Princeton University Press, Princeton, NJ, 1999.

[2] M. S. Baouendi, P. Ebenfelt, and L. P. Rothschild. Convergence and finite determination of formal CR mappings. J. Amer. Math. Soc., 13(4):697-723 (electronic), 2000.

[3] M. S. Baouendi, X. Huang, and L. P. Rothschild. Non-vanishing of the differential of holomorphic mappings at boundary points. Math. Res. Lett., 2:737-750, 1995.

[4] M. S. Baouendi and L. P. Rothschild. Germs of CR maps between real analytic hypersurfaces. Invent. math., 93:481-500, 1988.

[5] M. S. Baouendi and L. P. Rothschild. Geometric properties of mappings between hypersurfaces in complex space. J. Differential Geom., 31(2):473-499, 1990.

[6] M. S. Baouendi and L. P. Rothschild. A general reflection principle in $\mathbf{C}^{2}$. J. Funct. Anal., 99:409-442, 1991.

[7] M. S. Baouendi and L. P. Rothschild. A generalized complex Hopf lemma and its applications to CR mappings. Invent. math., 111:331-348, 1993.

[8] T. Bloom and I. Graham. On "type" conditions for generic real submanifolds of $\mathbb{C}^{n}$. Invent. Math., 40(3):217243, 1977.

[9] E.M. Chirka and Rea C. Normal and tangent ranks of CR mappings. Duke Math. J., 94:417-431, 1998.

[10] K. Diederich and J. E. Fornæss. Proper holomorphic mappings between real-analytic pseudoconvex domains in $\mathbb{C}^{n}$. Math. Ann., 282(4):681-700, 1988.

[11] P. Ebenfelt and L.P. Rothschild. Transversality of CR mappings. Amer. J. Math., (to appear).

[12] X. Huang. Schwarz reflection principle in complex spaces of dimension two. Comm. Partial Differential Equations, 21(11-12):1781-1828, 1996.

[13] X. Huang and Y. Pan. Proper holomorphic mappings between real-analytic domains in $\mathbb{C}^{n}$. Duke Math. J., 82:437-446, 1996.

[14] J. J. Kohn. Boundary behavior of $\bar{\partial}$ on weakly pseudo-convex manifolds of dimension two. J. Differential Geometry, 6:523-542, 1972.

[15] R. T. Kowalski. A hypersurface in $\mathbb{C}^{2}$ whose stability group is not determined by 2-jets. Proc. Amer. Math. Soc., 130(12):3679-3686 (electronic), 2002.

[16] R. T. Kowalski. Rational jet dependence of formal equivalences between real-analytic hypersurfaces in $\mathbb{C}^{2}$. Pacific J. Math., 220(1):107-139, 2005.

[17] B. Lamel and N. Mir. Finite jet determination of CR mappings. preprint, 2006.

[18] B. Lamel and N. Mir. Parametrization of local CR automorphims by finite jets and applications. J. Amer. Math. Soc, (to appear).

[19] F. Meylan. A reflection principle in complex space for a class of hypersurfaces and mappings. Pacific J. Math., 169(1):135-160, 1995.

[20] J.E. Fornæss. Biholomorphic mappings between weakly pseudoconvex domains. Pacific J. Math., 74:63-65, 1978.

[21] N. K. Stanton. Infinitesimal CR automorphisms of real hypersurfaces. Amer. J. Math., 118(1):209-233, 1996.

[22] D. Zaitsev. Unique determination of local CR-maps by their jets: A survey. Rend. Mat. Acc. Lincei, s. 9, 2002.

Universität Wien, Fakultät für Mathematik, Nordbergstrasse 15, A-1090 Wien, Österreich

E-mail address: lamelb@member.ams.org

Université de Rouen, Laboratoire de Mathématiques RaphaËl Salem, UMR 6085 CNRS, Avenue De L’Université, B.P. 12, 76801 Saint Etienne du Rouvray, France

E-mail address: Nordine.Mir@univ-rouen.fr 